\section{Social influences of} competition on impulsive choices in domestic chicks

\author{
Hidetoshi Amita, Ai Kawamori \\ and Toshiya Matsushima*
}

Laboratory for Animal Behaviour and Intelligence, Department of Biology, Faculty of Science, Hokkaido University, Sapporo, Japan *Author for correspondence (matusima@sci.hokudai.ac.jp).

Social factors involved in the control of impulsiveness were examined in domestic chicks. In binary choices between a large/long-delay option (LL) and a small/short-delay alternative (SS), chicks that had been competitively trained in groups of three individuals showed fewer choices of LL than did those trained in isolation (experiment 1), suggesting that competition causes impulsive choice. In experiment 2 , in order to identify the critical factor involved, we tested the effects of perceived competition (coincident feeding without interruption) and scrounging (gaining food without pecking bead) separately. To examine the effects of risk/noise that individual chicks experienced in competition, the food amount varied randomly in trials according to a binomial distribution around the expected mean. Perceived competition primarily contributed to the influence on the impulsive choice, whereas the contribution of scrounging was weaker. Collection risk did not explain the social influences since the perceived competition was not accompanied by actual interruption of the delayed food reward. The risk owing to variable food per se did not cause impulsive choices. Coincident foraging during competition is thought to play a critical role.

Keywords: temporal discounting; choice; risk; social foraging

\section{INTRODUCTION}

Animals often choose an immediate small reward even though a delayed alternative option yields a larger gain (Kalenscher \& Pennarts 2008; Matsushima et al. 2008). Since this behavioural trait clearly characterizes impulsiveness, measured in terms of high temporal discounting, relevant neural and developmental factors have been intensively studied in animals and humans (Cardinal 2006; Tripp \& Wickens 2008). It has been suggested that the reward is discounted because when there is a longer delay, there is a higher risk of being interrupted and consequently losing the reward ('collection risk' hypothesis; McNamara \& Houston 1987; Benson \& Stephens 1996; Sozou 1998). Interception of food items by competitive foragers is thought to be a major cause of the interruption. Although this argument is intuitively simple and

Electronic supplementary material is available at http://dx.doi.org/10. 1098/rsbl.2009.0748 or via http://rsbl.royalsocietypublishing.org. plausible, the link between competition and impulsiveness has not been empirically supported. A recent behavioural study in jays (Henly et al. 2008) failed to support the collection risk hypothesis, but the interruption was an experimentally incorporated termination of the task and was not caused by competitive foragers. It is, however, not so easy to study the effects of competition because competitive foraging can give rise to a complex set of factors including; (i) risk owing to the increased variability of food amount that each individual actually gains, and/ or (ii) accompanying noise (or decreased perceptual accuracy) owing to the increased variability. In competition, the amount of gained food would inevitably vary in every occasion. Foragers may thus undervalue the larger alternative because the longer delay increases the risk. Otherwise, a delay causes an increase in noise and the larger alternative is wrongly judged as being smaller. Alternatively, competition could affect choices through (iii) increased chance of gaining food without attentive foraging (scrounging); and (iv) presence of coincidently foraging individuals (perceived competition). Here, we present behavioural evidence that competitive foraging facilitates impulsive choice through perceived competition.

\section{MATERIAL AND METHODS}

A total of 105 male chicks (Gallus domesticus, white leghorns) were trained, but some chicks were discarded because they did not eat the millet food and tried to escape from the chamber, emitting distress calls. The present study is thus based on data obtained from 82 successfully trained individuals. See electronic supplementary materials for information on animals, apparatus for behavioural study, procedures and statistical analysis.

\section{(a) Experiment 1}

Chicks were trained to peck at coloured beads to gain delayed food reward. A blue bead was associated with a large/long-delay option (LL, six grains delivered after a delay of $0.0,1.5$ or $3.0 \mathrm{~s}$ ) and a red bead was associated with a small/short-delay alternative (SS, one grain after a delay of $0.0 \mathrm{~s}$ ). A white bead was used as a non-rewarding bead $\left(S_{-}\right)$. Chicks were trained for 3 days from post-hatch day 5 to 7 either in isolation (no competition) or in a group of three individuals (competition) (figure $1 a$ ). Nine chicks (three cages) were trained for each of the six groups. It is noticeable that the food was shared by the competing individuals, and those that pecked the bead did not necessarily gain all of the grain. Although we did not record the amount of food that each individual gained, we assumed that a longer delay to LL did not lead to a higher probability of interception of food by the accompanying competitors, as the interception occurred after the end of the delay period.

On day 8 , chicks in both groups were trained in isolation. Chicks were trained in one block of training per day, consisting of 72 pseudorandomly arranged trials with inter-trial intervals of 15-20 s: 18 trials with $\mathrm{LL} / S_{-}, 18$ trials with $\mathrm{SS} / S_{-}$and 36 trials with $S_{-}$/ $S-. S-$ means the non-rewarding white bead. On day 9 , in order to examine any lasting effects of competition on choices, chicks were individually tested in 20 trials with LL and SS. Contextual facilitation was disregarded since the chicks were tested singly. Test trials were randomly intermixed with $10 \mathrm{LL} / \mathrm{LL}$ and $10 \mathrm{SS} / \mathrm{SS}$ trials and 40 $S-/ S-$ trials. The side of presentation was counter-balanced among trials in each block. Most-likely-fitting curves were obtained by assuming a logistic model and a linear predictor. To statistically access the difference among groups, a generalized linear mixed model with AIC (Akaike's Information Criterion) was adopted.

\section{(b) Experiment 2}

Chicks were trained and tested according to the same timetable and colour assignment as in experiment 1 . To separate the factors (risk and/or noise, scrounging and perceived competition), we modified the experimental apparatus as shown in figure $2 a$. We assumed that each chick has an equal chance to get each grain in the competition condition, and set the amount to vary at every trial according to a binomial distribution in all groups (figure $2 b$ ). For SS, one grain was supplied in 18 trials and no food was supplied in the remaining 


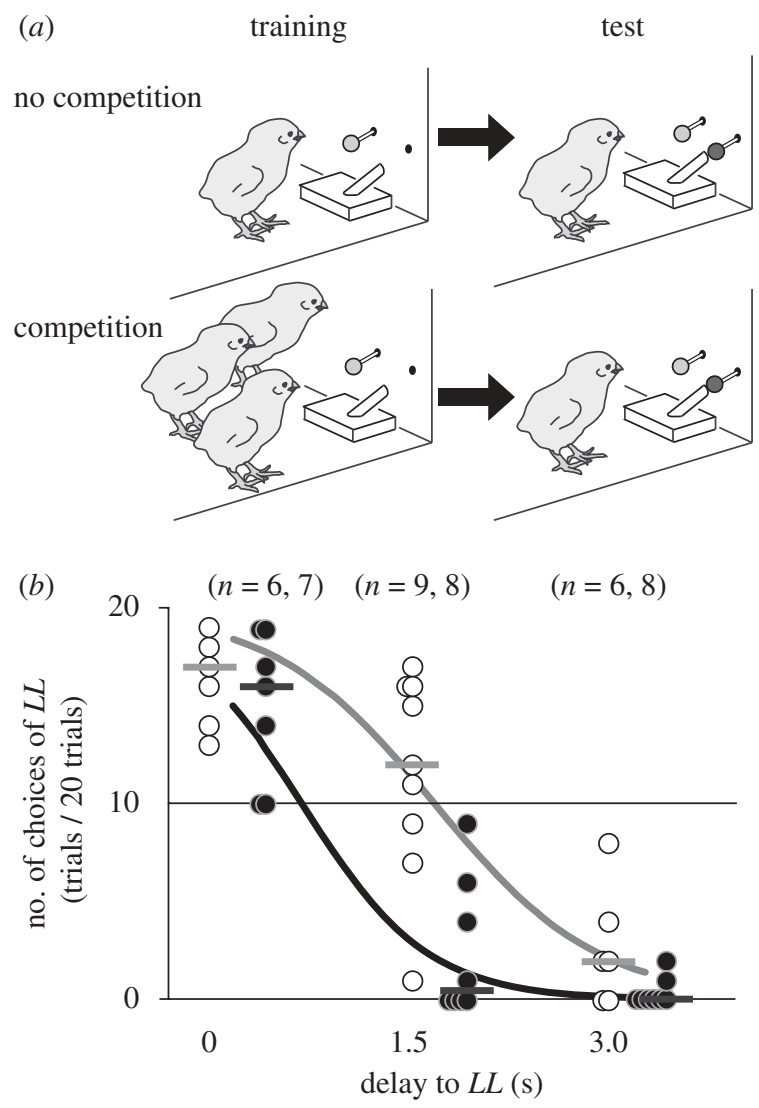

Figure 1. When trained competitively, chicks showed fewer choices of large/long delay reward (LL) over small/shortdelay reward (SS). (a) Experimental configuration of two groups of chicks-no competition and competition. (b) Number of choices of LL was plotted against delay of LL $(0,1.5$ or $3 \mathrm{~s})$ in six groups of chicks. Open and filled circles denote no competition and competition individuals respectively, and short horizontal bars indicate the median in each group. Most-likely-fitting curves are shown separately for competition and no competition groups.

36 trials, so that the mean was $1 / 3$ grain in 54 trials. These 54 trials were randomly assigned to three blocks of training on post-hatch day 5-7. For LL, similarly, 0-6 grains were supplied and the mean was set at two grains in 54 trials. Note, however, that the six-grain trial was not included because of its extremely low proportion. On day 8 (training in isolation) and day 9 (test), one grain and six grains were given for SS and LL, respectively.

To determine the contributions of two other factors (perceived competition ( $p c$ ) and scrounging $(s c)$ ), we compared choices of four groups arranged in a $2 \times 2$ factorial design (figure $2 c$ ). Twelve to fifteen chicks ( $4-5$ cages) were trained for each of the four groups. As in experiment 1 , we discarded chicks that were not trained. In the two groups (risk and risk $+p c$ ), chicks were trained without or with companion chicks placed in the left partition. The pair of companion chicks (scroungers) was supplied with three grains of millet at the time when the food was given to the subject. In the other two groups $($ risk $+s c$ and $r i s k+p c+s c)$, besides the training block given to risk and $p c$, two additional blocks of scrounging as a companion chick was given. Note that the scroungers competed for three grains and they were supposed to have experienced competition against each other.

\section{RESULTS}

Competition influenced impulsive choices (experiment 1). Based on the behavioural results (figure 1b), AICs were calculated for each of the eight models in which the factor of delay and competition was taken into account, together with their interactions; see table S1 of electronic supplementary material for details. Of these models, the delay-and-interaction model yielded the smallest AIC (111.0), whereas the delaycompetition-and-interaction model (full model) had the second smallest AIC (111.5). We therefore conclude that the competitive foraging modified choices in a manner dependent on the delay. In the delayand-interaction model, both coefficients of the delay and the interaction were estimated to be negative $\left(\beta_{2}=-1.13 \pm 0.23\right.$ and $\beta_{4}=-1.19 \pm 0.29$, respectively; estimate \pm s.e.), indicating that (i) an increase in the delay caused chicks to choose LL less frequently, and (ii) stronger effect of the delay was found in competition than in no competition.

Perceived competition was the primary factor involved in the social influences (experiment 2). AICs calculated based on the behavioural results (figure $2 c$ ) revealed that the competition-scrounging-and-interaction model (full model) yielded the smallest AIC (78.4), whereas the additive model had the second smallest AIC (96.8). In the full model, estimated coefficients of $p c$ and $s c$ were negative $\left(\gamma_{2}=-2.97 \pm 0.37\right.$ and $\gamma_{3}=-1.89 \pm$ 0.34 , respectively), indicating that (i) involvement of either factor made chicks choose LL less frequently, and (ii) the influence of perceived competition exceeded that of scrounging (see electronic supplementary material for the statistical analysis). The estimated coefficient of interaction was positive $\left(\gamma_{4}=+2.45\right)$, indicating that (iii) scrounging counteracted the influence of perceived competition.

On the other hand, variable food amount per se influenced choices in favour of LL. Chicks of the risk group in experiment 2 showed a significantly larger number of the choices of LL (median $=17$ ) than the chicks of the no competition group at $1.5 \mathrm{~s}$ in experiment 1 (median $=12)$; Mann-Whitney's $U$-test, $\mathrm{Ucal}=13.5, n_{1}=n_{2}=9, p=0.0179$.

\section{DISCUSSION}

The 'collection risk' hypothesis does not explain the observed influences of competition on impulsive choices, because perceived competition influenced behaviour without actual interruption of food. Variable food amount (and the accompanying risk and noise) also failed to account for the influence on the choices. In the training, chicks of the risk group (experiment 2) often failed to reliably peck at the bead associated with SS, suggesting that the low and variable profitability of the SS food led to a low subjective value. This is in agreement with our recent finding that chicks are risk-averse to variable amounts of food, particularly when the risky option includes non-rewarding trials (A. Kawamori \& T. Matsushima 2009, unpublished data). Alternatively, the impulsiveness could be owing to a higher level of perceptual noise, similar to that reported in risk-taking behaviours (Shafir et al. 2008). However, this hypothesis does not explain the observed difference between risk and risk $+p c$ in experiment 2 .

Some factor, other than the risk and noise, is hypothesized to be involved in the impulsive choices. Factors associated solely with the food amount might not be affected, since the choices of LL were similar in both 


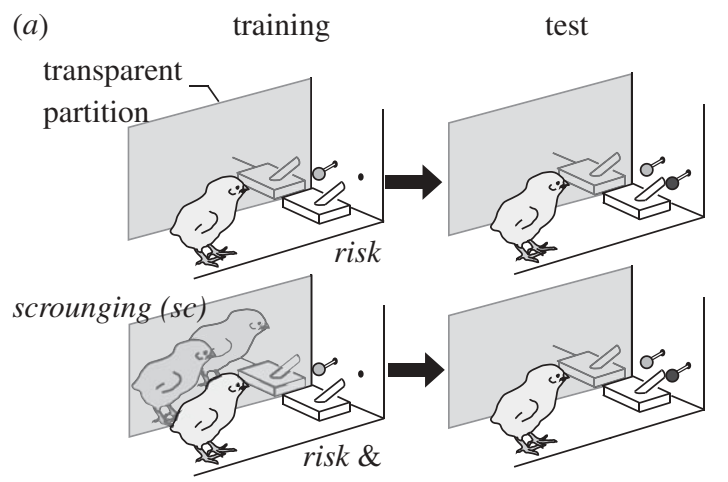

(b) perceived competition $(p c)$
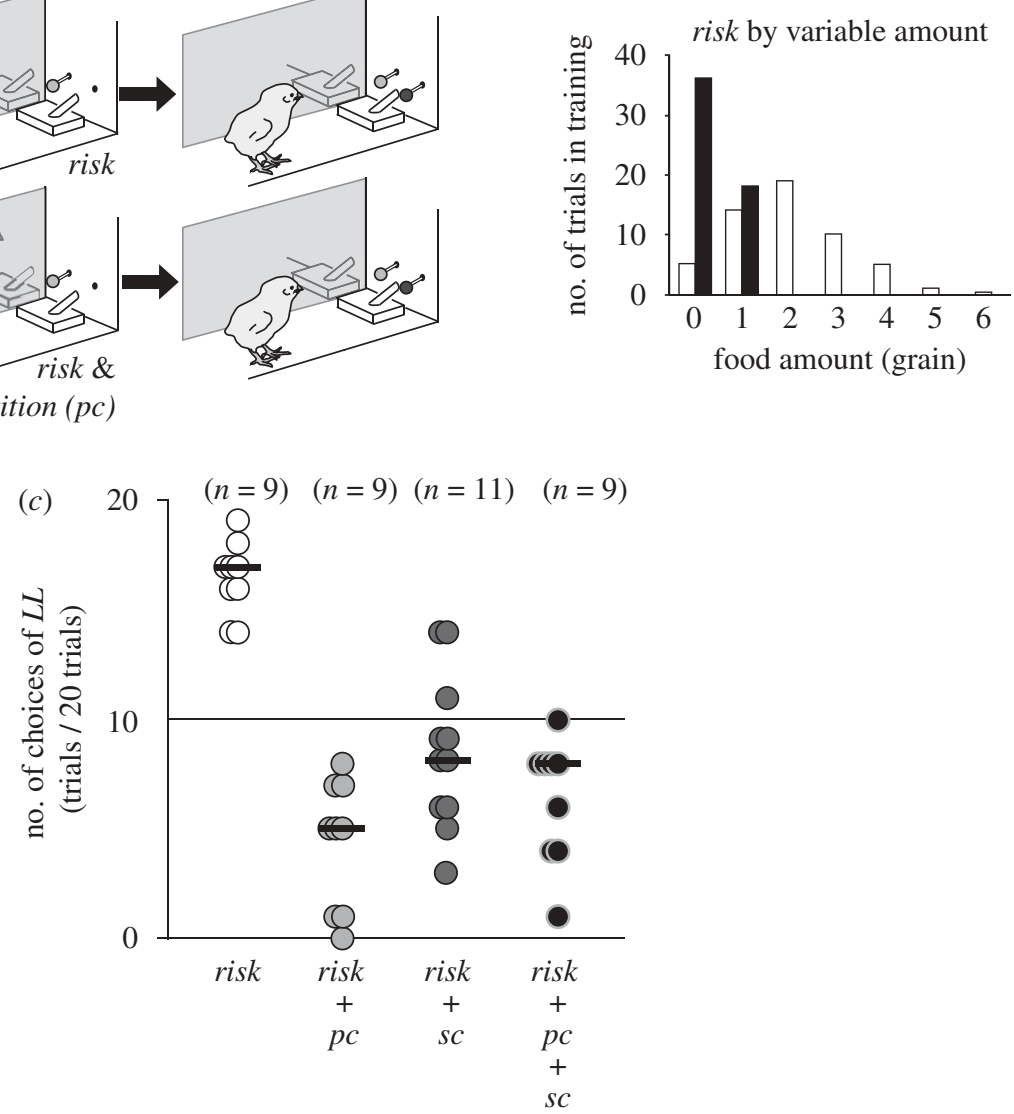

Figure 2. Perceived competition influenced impulsiveness without actual interception of food. (a) Experimental configuration of four groups of chicks, risk, risk $+p c$ (perceived competition), risk $+s c$ (risk and two additional blocks of scrounging, sc) and risk + $p c+s c$ (risk combined with $p c$ plus two blocks of $s c$ ). (b) Risk was experimentally introduced in terms of variable amount of food in each trial. Food amount was varied according to binomial distributions that were arranged to yield mean $=1 / 3$ grain (SS, filled columns) and two grains (LL, open columns), respectively. (c) Numbers of choices of LL were compared among four groups of chicks. Single symbols denote individual chicks, and horizontal bars indicate the median.

of the competition and no competition groups for the delay to $\mathrm{LL}=0 \mathrm{~s}$ (figure $1 b$ ). Of other possible factors involved, we examined the effects of perceived competition and scrounging separately. In the $p c$ and $p c+s c$ groups, chicks were successfully trained to peck at the SS bead, suggesting that the perceived competition increased the subjective value of SS, the immediate food. In this study, we did not know whether the subjective value of LL was decreased. The counteracting influence of scrounging could be ascribed to the fact that the scroungers gained the same amount of food (three grains) in both of the SS and the LL trials, thus obscuring the difference between these two options.

This study provides, to the best of our knowledge, the first empirical evidence of the lasting influence of social foraging (for reviews see Galef \& Giraldeau 2001) on economic decision-making (Kalenscher \& Pennarts 2008). In the context of social learning, it is often argued that animals learn what and where to forage (and even how to forage) in terms of response facilitation and local enhancement (Hoppitt \& Laland 2008). In this study, however, we did not dissociate these possible processes. Does the perceived competition influence impulsiveness even when the two feeders are placed at a distance? What happens if free food is given to the competitors not coincidentally with the subject? Cognitive processing underlying the effects of perceived competition on foraging decisions remains to be specified.

Experiments were conducted under guidelines and approval of the Committee on Animal Experiments of Hokkaido University. The guidelines are based on the national regulations for animal welfare in Japan (Law of the Humane Treatment and Management of Animals after a partial amendment no. 68, 2005).

The present study was supported by a grant from MEXT Japan (priority area on 'Mobiligence'; no. 20033001) and from JSPS (no. 19500260) to T.M. We express our sincere gratitude to the anonymous referees for their helpful suggestions.

Benson, K. E. \& Stephens, D. W. 1996 Interruptions, tradeoffs, and temporal discounting. Am. Zool. 36, $506-517$.

Cardinal, R. N. 2006 Neural systems implicated in delayed and probabilistic reinforcement. Neural Netw. 19, 1277-1301. (doi:10.1016/j.neunet.2006.03.004)

Galef, B. G. \& Giraldeau, A. 2001 Social influences on foraging in vertebrates: causal mechanism and adaptive functions. Anim. Behav. 61, 3-15. (doi:10.1006/anbe. 2000.1557) 
Henly, S. E., Ostdiek, A., Blackwell, E., Knutie, S., Dunlap, A. S. \& Stephens, D. W. 2008 The discounting-byinterruptions hypothesis: model and experiment. Behav. Ecol. 19, 154-162. (doi:10.1093/beheco/arm110)

Hoppitt, W. \& Laland, K. N. 2008 Social processes affecting feeding and drinking in the domestic fowl. Anim. Behav. 76, 1529-1543. (doi:10.1016/j.anbehav. 2008.07.011)

Kalenscher, T. \& Pennarts, C. M. A. 2008 Is a bird in the hand worth two in the future? The neuroeconomics of intertemporal decision-making. Prog. Neurobiol. 84, 284-315.

Matsushima, T., Kawamori, A. \& Bem-Sojka, T. 2008 Neuro-economics in chicks: foraging choices based on amount, delay and cost. Brain Res. Bull. 76, 245-252. (doi:10.1016/j.brainresbull.2008.02.007)
McNamara, J. M. \& Houston, A. I. 1987 A general framework for understanding the effects of variability and interruption on foraging behavior. Acta Bioltheor. 36, 3-22. (doi:10.1007/BF00159228)

Shafir, S., Reich, T., Erev, E. \& Lotem, A. 2008 Perceptual accuracy and conflicting effects of certainty on risktaking behavior. Nature 453, 917-920. (doi:10.1038/ nature06841)

Sozou, P. D. 1998 On hyperbolic discounting and uncertain hazard rates. Proc. R. Soc. Lond. B 265, 2015-2020. (doi:10.1098/rspb.1998.0534)

Tripp, G. \& Wickens, J. R. 2008 Dopamine transfer deficit: a neurobiological theory of altered reinforcement mechanisms in ADHD. F. Child Psychol. Psychiat. 49, 691-704. (doi:10.1111/j.1469-7610.2007.01851.x) 